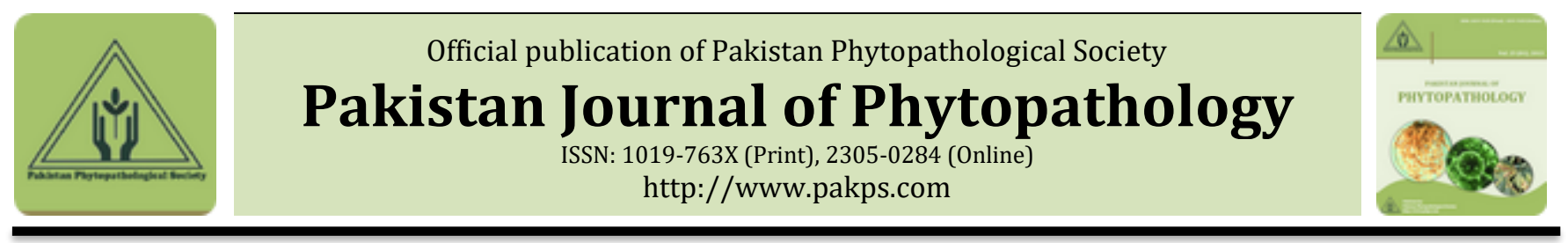

\title{
FUNGICIDAL MANAGEMENT OF ASSOCIATED MYCOFLORA WITH STORED SEEDS OF WHEAT AND CHICKPEA
}

\author{
Majid Kareem, Rashida Perveen, Syed A. H. Naqvi* \\ Department of Plant Pathology, Bahauddin Zakariya University, Multan, Pakistan.
}

\begin{abstract}
A B S T R A C T
Wheat and chickpea are considered the most important sources of food and energy and stored for longer period of time in the rural areas in the farmers sheds as seed for the next crop and as market commodity to sale. Both of these crops are liable to many mycoflora which not only deteriorate their quality but also leads toward the crop failure during the coming season. A number of seed borne pathogens viz., Aspergills niger, Aspergillus flavus, Aspergillus sulphureus, Aspergillus nidulense, Rhizopus stolonifer, Penicillium spp. and Drechslera spp, were isolated from wheat seed samples while Aspergillus niger, Aspergillus flavus, Bipolaris spp., Penicillium spp. and Rhizopus stolonifer were isolated from seed samples of chickpea. Various broad spectrum fungicides were evaluated against the isolated mycoflora which showed significant result. Score, Topsin-M and ridomil gold to be the most significant fungicides at all doses against the isolated mycoflora.
\end{abstract}

Keywords: Fungi, Fungicides, Wheat, Chickpea, Storage, Grains

\section{INTRODUCTION}

Wheat (Triticum aestivum) is an important cereal crop which belongs to family poaceae. Wheat is a rich source of proteins and carbohydrates and it also contains vitamins like riboflavin, thiamin, niacin, vitamin $E$ and minerals (P, Mg, Cu and Zn) (Adsule and Kadam, 1986). The area under cultivation of wheat is about 9.49 million ha with 26.52 million tonnes production (FAO, 2007). Over $70 \%$ of the gross cereals and over $36 \%$ of the country's acreage is devoted to cultivation of wheat. Wheat crop is being cultivated in our country from ancient times. According to some experts wheat was first cultivated in the Indus Valley. Pakistan is the 4th largest producer of wheat in Asia and stands 11th in world production (Govt. of Pakistan, 2014).

Chickpea (Cicer arietinum L.) is an important pulse crop belonging to Leguminose family, ranking third after dry beans (Phaseolus vulgaris L) and dry peas (Pisum

\footnotetext{
Submitted: January 03, 2018

Revised: June 06, 2018

Accepted for Publication: August 01, 2018

* Corresponding Author:

Email: atifhnaqvi@bzu.edu.pk

(C) 2017 Pak. J. Phytopathol. All rights reserved.
}

sativum L) (Dhar and Gurha, 1998). The Desi and Kabuli chickpea is cultivated throughout the world with different names i.e., Chickpea (UK), Bengal gram (Indian), Garbanzo (Latin America), Shimbra (Ethiopia), Hommes, Hamaz (Arab world) and Nohud and Loblebi (Turkey). Chickpea is self-pollinated crop, up to $1 \%$ cross pollinated (Smithson et al., 1985). Pakistan ranked second in the world in area and third in production of chickpea. Annual production is about 760 thousand ton from an area of 1094 thousand hectares and $4.7 \%$ shares in national economy (Govt. of Pakistan, 2014). Chickpea is cultivated on $82 \%$ area is in Punjab, 9\% NWFP, $8 \%$ Sindh and $1 \%$ Baluchistan whereas $90 \%$ is cultivated in rain fed areas in Punjab. Thal region is the home of chickpea considered as major chickpea producing area in Pakistan and it produces $80 \%$ of chickpea (Khan et al., 1991). Chickpea has high nutritional value and its edible grains contain proteins, vitamins, carbohydrates and minerals along with $25.3-28.9 \%$ protein contents (Hulse, 1994). In addition to proteins, it has fiber $3 \%$, carbohydrates $35-59 \%$, ash $3 \%$, calcium $0.2 \%$, oil 4.8 $5.5 \%$ and phosphorus $0.3 \%$. Its proteins digestibility is about $76-78 \%$ and carbohydrates digestibility near about $57-60 \%$. 
Plant pathogens are transported by seeds over long distances. There are many examples in agriculture literature that infected or contaminated seeds with pathogens cause the spread of plant diseases (Sinclair and Agarwal, 1997). Loose smut (Ustilagonuda tritici), karnal bunt (Tillettiainidica), head blight or scab (Fusarium spp.), tundo or ear cockle (Clavibacter tritici and Anguina tritici) are major seed borne diseases of wheat crop that reduces the crop yield and grain quality (Kumar et al., 2008). Major diseases of wheat are transmitted by fungal infected seeds. Seed borne pathogens includes Aspergillus, Penicillium spp., Helmithosporium sativum, Fusarium graminearum, Alternaria tenuis, Drechslera sorokiniana, $D$. tetramera, Cladosporium oxysporum and Curvularia lunata (Bhutta and Hussain, 1999). Seed borne and storage fungi of wheat recently reported include Drechslera sorokiniana, Alternaria alternate, Fusarium moniliforme, F.graminearum, F. avenacium, $F$. culmorum, F. equiseti, F. nivale, F. sporotirchioides, Stemphylium botryosum, Cladosporium herbarum (How much recent? Ref.) Near about $20 \%$ of wheat every year lost due to diseases (Fakir, 1999). Seed health is very important and it plays a key role for high yield and successful cultivation. Seed borne fungi are most important factor that affects health of seed. Infected seeds reduce seed germination which results in lower crop production. Healthy seeds play a vital role in high yield and successful cultivation of crop. Seed borne mycoflora of wheat is responsible for lower yield up to $15-90 \%$ if untreated seeds are sown in field (Wiese, 1984).

Many fungal pathogens viz., Alternaria alternata, Alternaria porri, Aspergillus amstelodami, $A$. fumigatus, A. flavus, A. nadulans, A. niger, A. sydowi, Cladosporium maccrocarpum, Botrytis cinerea, Macrophominia phaesolina, Fusarium equiseti, $F$. oxysporum, $F$. moniliforme, $F$. semitectum, Penicillium notatum, Rhizoctonia spp., Myrothecium roridum and Rhizopus arhizus reported from chickpea (Ahmad et al., 1993). Ascocyta blight caused by Ascocyta rabiei (Nene, 1980), Botrytus grey mould caused by Botrytus cinerea appeared in the form of grey to brown lesion (Joshi and Singh, 1969). Colletotrichum blight caused by Colletotrichum dematium, Alternaria blight is caused by Alternaria alternate (Vishwakarima and Chaudhary, 1974). If untreated seeds are sown in field then seed borne fungi cause yield loss about $15-90 \%$ and it also affect the plant morphology (Wiese, 1984). Health of seed is very important for good quality of seed. Serious disease outbreaks are the results of seed dissemination of plant pathogens. Infected seeds affect the grain yield and quality by producing abnormal seedlings and lower germination (Bishaw et al., 2013). Seed borne fungi are responsible for seedling damage, seed rot, seed abortion and cause the systemic or local infection (Ryley et al., 1989). Each year about $20 \%$ of wheat is lost due to diseases (Fakir, 1999). Dharmvir et al., (1968) reported storage fungi cause $42 \%$ reduction in plant population. Seed borne diseases cause the $12 \%$ global losses of potential production. Keeping in view the importance of seed health in seed storage the current research was planned to isolate and identify the associated mycoflora with stored grains and to evaluate various broad spectrum fungicides against isolated mycoflora under in-vitro.

\section{MATERIALS AND METHODS}

Study site: The current research was conducted at the Department of Plant Pathology, Faculty of Agricultural Sciences and Technology, Bahaudin Zakariya University, Multan.

Collection of seed samples: A total of 75 seed samples of wheat half kilogram each was collected from farmer's stored seeds at District Layyah located in southern Punjab region. The District Layyah consists of three Tehsils like Tehsil Layyah, Karor Lal Esan and Tehsil Chobara. In each Tehsil 25 farmers were randomly selected and seed samples were collected. A total of 75 seed samples of chickpea were collected from three tehsils of District Layyah but mostly seed samples of chickpea were collected from Tehsil Chobara because it is rain fed area and is very famous for chickpea growing and cultivation. Half kg seed samples collected from each selected farmer and were packed in polythene bags labeled and stored at room temperature for further processing and assay of seed-borne pathogens.

Isolation of seed borne fungi: Doyer (1938) developed the standard blotter method and was included in the International Seed Testing Association Rules of 2005 used in the identification of seed- borne fungal pathogens in this study. Fifteen seeds of each sample were tested in 3 replications. 
Three pieces of $9 \mathrm{~cm}$ sized blotting papers were moistened with distilled water and placed in $9 \mathrm{~cm}$ autoclaved petri dishes. After draining the excess water, treated seeds at the rate of 5 seeds per petri dish were placed at equal distance. The plates were incubated for 7 days at $25^{\circ} \mathrm{C}$.

Identification of seed borne pathogens: Fungi was isolated by blotter method and sterile PDA and incubated for 7 days. After that the fungi were identified based on their colony colour, spore morphology and type of mycelia growth using the microscope. The associated fungi and they were identified based on "habit and colony characters in culture. Fungal species found growing on the surface of seeds were identified and their percentage of infection frequency of occurrence was calculated using the following formula:

$$
\text { Infection frequency }=\frac{\text { No. of infected seeds }}{\text { Total No. of seeds }} \times 100
$$

Evaluation of various fungicides against the isolated mycoflora through poison food technique: Most commonly practiced method is the poison food technique for evaluating fungicides under laboratory conditions. Fungicides were mixed in PDA and then PDA was poured into autoclaved petri plates and $1 \mathrm{~cm}$ disk of the culture was placed in the center of the Petri dish and incubated at $25^{\circ} \mathrm{C}$ temperature. Eight fungicides namely Triton, Alliete, Ridomil gold, Nativo, Score, Mancozeb, Topsin-M and Hexaconazole were used to determine the mycilial growth of different isolated fungi (Table 1). Each fungicide were used at the rate of $01 \mu \mathrm{g} \mathrm{ml}-1,10 \mu \mathrm{g}$ ml-1, $25 \mu \mathrm{g}$ ml-140 $\mu \mathrm{g} \mathrm{ml-1and} 60 \mu \mathrm{g} \mathrm{ml}-1$ were used for poison food technique during laboratory study. Different concentration of fungicidal suspension was prepared by dissolving the desired quantities of all fungicides in warm PDA. After autoclaving PDA, fungicides were mixed and incorporated completely in the medium by shaking with the hands. About $15 \mathrm{ml}$ of fungicidal medium was poured into each $9 \mathrm{~cm}$ sized autoclaved petri plate. After solidification $1 \mathrm{~cm}$ bit of isolated culture of fungi were placed on petri dishes containing fungicidal medium. Three replications were used for each concentration of every fungicide. Three petri dishes without fungicides were used as a control. After this they were incubated at $25^{\circ} \mathrm{C}$ for 7 days. Petri plates were measured from bottom side for mycelial growth of PDA with and without fungicides (Table 1).

Table 1. List of fungicides tested against in vitro mycelial growth of various isolated mycoflora from wheat and chickpea.

\begin{tabular}{|c|c|c|c|c|c|c|}
\hline Trade Name & Active ingredient & Action & a.i* $(\%)$ & Formulation & Toxicity class & Manufacturer \\
\hline Alliete ${ }^{\circledR}$ & FostylAluminium & S & 80 & WP & III & Bayer Crop Science \\
\hline Ridomil Gold $^{\circledR}$ & Metalaxyl + Mancozeb & $\mathrm{C}$ & 4 & WG & IV & Syngenta \\
\hline Nativo $^{\circledR}$ & Tebuconazole + Trifloxystrobin & $\mathrm{S}$ & 50 & WG & III & Bayer crop science \\
\hline Score ${ }^{\circledR}$ & Difenoconazole & $S$ & 24 & $\mathrm{EC}^{*}$ & III & Syngenta \\
\hline Topsin- $\mathrm{M}^{\circledR}$ & Thiophanate-methyl & $S$ & 70 & WP & III/IV & Arysta Life Sciences \\
\hline Contaf Plus ${ }^{\circledR}$ & Hexaconazole & $\mathrm{S}^{*}$ & 5 & SC & III & Jaffer Agro Services \\
\hline
\end{tabular}

*a.i: active ingredient, ${ }^{* W P}$ : wettable powder, ${ }^{*}$ EC: emulsifiable concentrate, ${ }^{*} \mathrm{C}$ : contact, ${ }^{*}$ : systemic, ${ }^{*}$ WG: wetable granules, ${ }^{*}$ SC: suspension concentrate, ${ }^{*}$ SL: soluble liquid.

\section{RESULTS}

Isolation of fungi associated with stored seeds of wheat and chickpea: A number of seed borne fungal pathogens were isolated from seed samples of chickpea and wheat. It was observed that Aspergills niger, Aspergillus flavus, Aspergillus sulphureus, Aspergillus nidulense, Rhizopus stolonifer, Penicillium spp and Drechslera spp were isolated from wheat seed samples. Isolation results showed that maximum infection frequencies were Aspergillus flavus, Aspergillus niger,
Rhizopus stolonifer and Penicillium spp from seed samples of wheat. Minimum frequencies of Drechslera spp, Aspergillus nidulense and Aspergillus sulphureus were recorded from wheat seed samples. While Aspergillus niger, Aspergillus flavus, Bipolaris spp, Penicillium spp and Rhizopus stolonifer were isolated from 3 seed samples of chickpea. Maximum infection frequencies were showed by Aspergillus niger, Aspergillus flavus and Penicillium spp, but minimum frequency were Bipolaris spp from chickpea samples (Table 2). 
Table 2. Isolation frequency of mycoflora associated with wheat and chickpea stored grains.

\begin{tabular}{llll}
\hline S. No. & Type of sample & Fungi associated & Infection frequency \% \\
\hline 1. & Wheat & Aspergillus niger & 89.50 \\
& Aspergillus flavus & 91.85 \\
& Aspergillus sulphureus & 33.23 \\
& Aspergillus nidulense & 26.63 \\
& Rhizopus stolonifer & 83.42 \\
& Penicillium spp. & 73.67 \\
& Drechslera spp. & 13.33 \\
\hline 2. & Aspergillus niger & 71.37 \\
& Chickpea & Aspergillus flavus & 66.67 \\
& Bipolaris spp. & 23.69 \\
& & Penicillium spp. & 67.34 \\
\hline
\end{tabular}

Evaluation of various fungicides against the isolated mycoflora through poison food technique: The results of current study suggested that among the eight systemic fungicides (Table 1) evaluated against Aspergillus flavus, score (difenoconazole) was significantly superior to all other fungicides with mycelial growth of $2.33,3.77,5.57,7.30,8.73 \mathrm{~cm}$ at 60 , $40,25,10,01 \mu \mathrm{g} \mathrm{ml}^{-1}$ concentration, respectively. After this, Topsin-M (thiophanate-methyl) showed the good results and these were $2.77,4.63,5.60,7.90,8.53 \mathrm{~cm}$ at similar concentrations. In case of alliete (fostyl aluminium) and Dithane m-45 (mancozeb), there was a significant decrease in colony diameter at tested doses as compared to control. Contaf plus (hexaconazole) was found to be the least effective at similar concentrations. Triton (validamycin) was the least effective among all the fungicides tested against Aspergillus flavus (Table 3). While among the eight fungicides against Aspergillus niger, topsin-m (thiophanate-methyl) was significantly superior to all other fungicides with mycelial growth of $2.03,3.60,5.67,7.23,8.80 \mathrm{~cm}$ at $60,40,25,10,01 \mu \mathrm{g} \mathrm{ml}^{-1}$ concentration, respectively. After topsin-m, score (difenoconazole) showed the better results with inhibition zone of mycelial growth with 2.30, 4.07, 6.03, $7.70,8.73 \mathrm{~cm}$ at similar doses. Ridomil gold (metalaxyl + mancozeb), alliete(fostyl aluminium) and also showed the good results against Aspergillusniger at similar concentration. Contaf plus (hexaconazole) was the least effective among all the eight fungicides tested with mycelial growth $3.80,6.05,7.40,8.10 \mathrm{~cm}$ at $60,40,25$, $10 \mu \mathrm{g} \mathrm{ml}^{-1}$ concentration against Aspergillus niger (Table 4). Similarly fungicides evaluated against Aspergillus sulphureus, score (difenoconazole) was significantly superior to all other fungicides with mycelial growth of $2.50,4.53,6.23,7.63,8.60 \mathrm{~cm}$ at $60,40,25,10,01 \mu \mathrm{g} \mathrm{ml}^{-1}$ concentration, respectively. After this, Ridomil gold (metalaxyl + mancozeb) showed the better results and these were $2.80,5.47,7.20,8.50,8.90 \mathrm{~cm}$ at similar concentrations. While nativo (tebuconazole + trifloxystrobin) showed the significant results against Aspergillus sulphureus, there was a significant decrease in colony diameter at 60, 40 and $25 \mu \mathrm{g} \mathrm{ml}-1$ tested doses as compared to control. Contaf plus (hexaconazole) was found to be the least effective at similar concentrations. Triton (validamycin) was the least effective among all the fungicides tested against Aspergillus sulphureus (Table 5). Likewise results showed that Aspergillus nidulens was best controlled by score (difenoconazole) and found to be significantly superior to all other fungicides with mycelial growth of $2.83,4.33,6.00,7.33$, $8.67 \mathrm{~cm}$ at $60,40,25,10,01 \mu \mathrm{g} \mathrm{ml}-1$ concentration, respectively. After score, topsin-m (thiophanate-methyl) showed the significant results with mycelial growth of $2.87,4.63,5.83,7.33,8.87 \mathrm{~cm}$ at similar doses. Nativo (tebuconazole + trifloxystrobin) also showed the good results against Aspergillus nidulens at similar concentration. Triton (validamycine) was the least effective among all the eight fungicides tested at similar concentrations against Aspergillus nidulense (Table 6).

Furthermore, score (difenoconazole) showed maximum results among all the eight systemic (differentiate the fungicides) fungicides evaluated against Rhizopus stolonifer with mycelial growth of $2.50,4.40,6.10,7.37,8.73 \mathrm{~cm}$ at $60,40,25,10,01 \mu \mathrm{g}$ $\mathrm{ml}^{-1}$ concentration, respectively. Contaf plus (hexaconazole) showed the significant results with inhibition zone of $2.97,4.50,7.87,8.47$ and $8.93 \mathrm{~cm}$ at similar concentrations. Topsin-m (thiophanatemethyl) and Dithane M-45 (mancozeb) also gave the better results against Rhizopus stplonifer at 
similar tested doses. While alliete (fostyl aluminium), nativo (tebuconazole + trifloxystrobin) and ridomil gold (metalaxyl + mancozeb) showed the least significant results (Table 7) whereas Penicillium, was best controlled by ridomil gold (metalaxyl + mancozeb) and proved significantly superior to all other fungicides with mycelial growth of 2.37, $4.10,6.97 \mathrm{~cm}^{2}$ at $60,40,25 \mu \mathrm{g} \mathrm{ml}^{-1}$ concentration, respectively. After ridomil gold, score (difenoconazole) showed the better results with inhibition zone of mycelial growth with 2.63, $4.37,6.03,7.33,8.57 \mathrm{~cm}$ at similar doses. Contaf plus (hexaconazole) was the least effective among all the eight fungicides tested with mycelial growth $4.00,6.67,7.63,8.30 \mathrm{~cm}$ at 60 ,
40, 25, $10 \mu \mathrm{g} \mathrm{ml}-1$ concentration against Penicillium. While Dithane M-45 (mancozeb) and alliete (fostyl aluminium) also showed least significant results (Table 8). The poison food technique results showed that Drechslera, was best controlled by score (difenoconazole) with mycelial growth of 2.50, 4.17, 6.07, 7.43, $8.53 \mathrm{~cm}$ at $60,40,25,10,01 \mu \mathrm{g} \mathrm{ml}-1$ concentration, respectively. After score, contaf plus (hexaconazole) gave the best result at highest concentration. Topsin-m (thiophanate-methyl) showed the significant results with mycelial growth of 2.93, 4.70, 5.70, 7.60, $8.70 \mathrm{~cm}$ at similar doses. Triton (validamycin) was the least effective among all the eight fungicides tested at similar concentrations against Drechslera (Table
9). Hence, Bipolaris was significantly controlled by topsin-m (thiophanate methyl) with mycelial growth of $2.67,4.10,5.20,7.30,8.67 \mathrm{~cm}$ at 60 , $40,25,10,01 \mu \mathrm{g} \mathrm{ml}^{-1}$ concentration, respectively. After this, score (difenoconazole) showed the better results and these were $2.80,4.10,5.73$, 7.37, $8.63 \mathrm{~cm}$ at similar concentrations. While Ridomil gold (metalaxyl + mancozeb) showed the better results against Bipolaris, there was a significant decrease in colony diameter at similar tested doses as compared to control. Contaf plus (hexaconazole) was found to be the least effective at similar concentrations. Triton (validamycin) was the least effective among all the fungicides tested against Bipolaris (Table 10).

Table 3. Effect of different fungicides on mycelial growth of Aspergillus flavus

\begin{tabular}{|c|c|c|c|c|c|c|c|c|}
\hline \multicolumn{9}{|c|}{ Mycelial growth $\left(\mathrm{cm} \pm \mathrm{S} . \mathrm{E}^{*}\right)$} \\
\hline Concentration $\left(\mu \mathrm{g} \mathrm{ml}^{-1 *}\right)$ & Triton & Alliete & Ridomil Gold & Nativo & Score & Mancozeb & Topsin-M & Hexaconazole \\
\hline $01 \mu \mathrm{g} \mathrm{ml}^{-1}$ & $8.93 \mathrm{ab} \pm 0.03$ & $8.53 \mathrm{a} \pm 0.13$ & $8.50 \mathrm{a} \pm 0.05$ & $8.90 \mathrm{a} \pm 0.09$ & $8.73 a \pm 0.08$ & $8.93 a \pm 0.06$ & $8.53 \mathrm{ab} \pm 0.28$ & $8.70 \mathrm{a} \pm 0.09$ \\
\hline $10 \mu \mathrm{g} \mathrm{ml}^{-1}$ & $8.27 b \pm 0.18$ & $8.17 \mathrm{a} \pm 0.43$ & $8.33 a \pm 0.26$ & $8.67 \mathrm{a} \pm 0.03$ & $7.30 \mathrm{~b} \pm 0.17$ & $7.60 \mathrm{~b} \pm 0.22$ & $7.90 \mathrm{~b} \pm 0.10$ & $8.17 a b \pm 0.06$ \\
\hline $25 \mu \mathrm{g} \mathrm{ml}^{-1}$ & $7.40 \mathrm{c} \pm 0.25$ & $6.47 b \pm 0.28$ & $7.47 \mathrm{~b} \pm 0.24$ & $6.93 \mathrm{~b} \pm 0.23$ & $5.57 \mathrm{c} \pm 0.15$ & $6.03 \mathrm{c} \pm 0.08$ & $5.60 \mathrm{c} \pm 0.23$ & $7.63 \mathrm{~b} \pm 0.13$ \\
\hline $40 \mu \mathrm{g} \mathrm{ml}^{-1}$ & $6.37 \mathrm{~d} \pm 0.32$ & $5.10 \mathrm{c} \pm 0.30$ & $4.63 c \pm 0.25$ & $5.37 \mathrm{c} \pm 0.25$ & $3.77 \mathrm{~d} \pm 0.12$ & $4.37 \mathrm{~d} \pm 0.25$ & $4.63 \mathrm{~d} \pm 0.21$ & $6.23 c \pm 0.29$ \\
\hline $60 \mu \mathrm{g} \mathrm{ml}^{-1}$ & 4.03 e \pm 0.12 & $3.00 \mathrm{~d} \pm 0.40$ & $3.37 d \pm 0.23$ & $2.93 \mathrm{~d} \pm 0.13$ & 2.33 e \pm 0.10 & 3.03 e \pm 0.06 & 2.77 e \pm 0.12 & $3.87 \mathrm{~d} \pm 0.48$ \\
\hline Control & $9.00 \mathrm{a} \pm 0.00$ & $8.90 \mathrm{a} \pm 0.09$ & $8.80 \mathrm{a} \pm 0.10$ & $8.97 \mathrm{a} \pm 0.03$ & $8.93 a \pm 0.03$ & $9.00 \mathrm{a} \pm 0.00$ & $8.77 a \pm 0.03$ & $8.87 \mathrm{a} \pm 0.08$ \\
\hline LSD* & 0.73 & 0.94 & 0.75 & 0.58 & 0.42 & 0.41 & 0.70 & 0.77 \\
\hline
\end{tabular}

Means followed by the same letters in each column are not statistically different $\left({ }^{*} \mathrm{P}<0.05\right)$ LSD* ${ }^{*}=$ Least significant difference, S.E* ${ }^{*}=$ Standard error, $\mu \mathrm{g}$ $\mathrm{ml}^{-1 *}=$ Micro gram per milliliter 
Table 4. Effect of different fungicides on mycelial growth of Aspergillus niger

\begin{tabular}{ccccccccc}
\hline & & \multicolumn{4}{c}{ Mycelial growth $\left(\mathrm{cm} \pm \mathrm{S} . \mathrm{E}^{*}\right.$ ) } \\
\hline Concentration $\left(\mu \mathrm{g} \mathrm{ml}^{-1 *}\right)$ & Triton & Alliete & Ridomil Gold & Nativo & Score & Mancozeb & Topsin-M & Hexaconazole \\
\hline $01 \mu \mathrm{g} \mathrm{ml}^{-1}$ & $8.70 \mathrm{a} \pm 0.10$ & $8.77 \mathrm{a} \pm 0.10$ & $8.73 \mathrm{a} \pm 0.13$ & $8.87 \mathrm{a} \pm 0.12$ & $8.73 \mathrm{a} \pm 0.08$ & $8.60 \mathrm{a} \pm 0.13$ & $8.80 \mathrm{a} \pm 0.13$ & $8.53 \mathrm{ab} \pm 0.03$ \\
\hline $10 \mu \mathrm{g} \mathrm{ml}^{-1}$ & $7.67 \mathrm{~b} \pm 0.10$ & $6.90 \mathrm{~b} \pm 0.40$ & $8.43 \mathrm{a} \pm 0.25$ & $8.20 \mathrm{a} \pm 0.22$ & $7.70 \mathrm{~b} \pm 0.22$ & $7.43 \mathrm{~b} \pm 0.24$ & $7.23 \mathrm{~b} \pm 0.21$ & $8.10 \mathrm{~b} \pm 0.10$ \\
\hline $25 \mu \mathrm{g} \mathrm{ml}^{-1}$ & $6.30 \mathrm{c} \pm 0.20$ & $5.83 \mathrm{~b} \pm 0.38$ & $7.17 \mathrm{~b} \pm 0.48$ & $6.47 \mathrm{~b} \pm 0.28$ & $6.03 \mathrm{c} \pm 0.10$ & $5.90 \mathrm{c} \pm 0.30$ & $5.67 \mathrm{c} \pm 0.13$ & $7.40 \mathrm{c} \pm 0.20$ \\
\hline $40 \mu \mathrm{g} \mathrm{ml}^{-1}$ & $4.80 \mathrm{~d} \pm 0.13$ & $4.33 \mathrm{c} \pm 0.43$ & $4.73 \mathrm{c} \pm 0.23$ & $4.53 \mathrm{c} \pm 0.36$ & $4.07 \mathrm{~d} \pm 0.20$ & $4.40 \mathrm{~d} \pm 0.22$ & $3.60 \mathrm{~d} \pm 0.18$ & $6.05 \mathrm{~d} \pm 0.46$ \\
\hline $60 \mu \mathrm{g} \mathrm{ml}^{-1}$ & $3.43 \mathrm{e} \pm 0.15$ & $3.07 \mathrm{~d} \pm 0.39$ & $2.83 \mathrm{~d} \pm 0.06$ & $3.30 \mathrm{~d} \pm 0.10$ & $2.30 \mathrm{e} \pm 0.10$ & $3.43 \mathrm{e} \pm 0.21$ & $2.03 \mathrm{e} \pm 0.06$ & $3.80 \mathrm{e} \pm 0.23$ \\
\hline Control & $8.93 \mathrm{a} \pm 0.06$ & $9.00 \mathrm{a} \pm 0.00$ & $8.87 \mathrm{a} \pm 0.06$ & $8.97 \mathrm{a} \pm 0.03$ & $9.00 \mathrm{a} \pm 0.00$ & $8.83 \mathrm{a} \pm 0.03$ & $9.00 \mathrm{a} \pm 0.00$ & $8.77 \mathrm{a} \pm 0.03$ \\
\hline LSD $^{*}$ & 0.48 & 1.25 & 0.81 & 0.83 & 0.51 & 0.63 & 0.46 & 0.52 \\
\hline
\end{tabular}

Means followed by the same letters in each column are not statistically different ( $* \mathrm{P}<0.05)$, LSD*= Least significant difference, S.E*= Standard error, $\mu \mathrm{g}$ $\mathrm{ml}^{-1 *}=$ Micro gram per milliliter

Table 5. Effect of different fungicides on mycelial growth of Aspergillus sulpherous

\begin{tabular}{|c|c|c|c|c|c|c|c|c|}
\hline \multirow[b]{2}{*}{ Concentration $\left(\mu \mathrm{g} \mathrm{ml}^{-1 *}\right)$} & \multicolumn{7}{|c|}{ Mycelial growth $\left(\mathrm{cm} \pm\right.$ S.E $\left.{ }^{*}\right)$} & \multirow[b]{2}{*}{ Hexaconazole } \\
\hline & Triton & Alliete & Ridomil Gold & Nativo & Score & Mancozeb & Topsin-M & \\
\hline $01 \mu \mathrm{g} \mathrm{ml}^{-1}$ & $8.93 \mathrm{ab} \pm 0.06$ & $8.83 a \pm 0.06$ & $8.90 \mathrm{a} \pm 0.09$ & $8.93 a \pm 0.06$ & $8.60 \mathrm{a} \pm 0.10$ & $9.00 \mathrm{a} \pm 0.00$ & $8.87 \mathrm{a} \pm 0.08$ & $8.83 \mathrm{a} \pm 0.10$ \\
\hline $10 \mu \mathrm{g} \mathrm{ml}^{-1}$ & $8.33 \mathrm{~b} \pm 0.13$ & $8.20 \mathrm{a} \pm 0.18$ & $8.50 \mathrm{a} \pm 0.20$ & $8.63 a \pm 0.08$ & $7.63 \mathrm{~b} \pm 0.08$ & $7.90 \mathrm{~b} \pm 0.10$ & $7.70 \mathrm{~b} \pm 0.05$ & $8.30 \mathrm{ab} \pm 0.10$ \\
\hline $25 \mu \mathrm{g} \mathrm{ml}^{-1}$ & $7.60 \mathrm{c} \pm 0.13$ & $6.70 \mathrm{~b} \pm 0.36$ & $7.20 \mathrm{~b} \pm 0.13$ & $6.83 b \pm 0.15$ & $6.23 c \pm 0.12$ & $6.00 \mathrm{c} \pm 0.05$ & $5.70 \mathrm{c} \pm 0.26$ & $7.70 \mathrm{~b} \pm 0.13$ \\
\hline $40 \mu \mathrm{g} \mathrm{ml}^{-1}$ & $6.70 \mathrm{~d} \pm 0.36$ & $5.03 \mathrm{c} \pm 0.35$ & $5.47 \mathrm{c} \pm 0.24$ & $5.10 \mathrm{c} \pm 0.10$ & $4.53 \mathrm{~d} \pm 0.29$ & $4.40 \mathrm{~d} \pm 0.28$ & $4.70 \mathrm{~d} \pm 0.10$ & $6.17 \mathrm{c} \pm 0.47$ \\
\hline $60 \mu \mathrm{g} \mathrm{ml}^{-1}$ & $4.27 \mathrm{e} \pm 0.10$ & $3.57 \mathrm{~d} \pm 0.15$ & $2.80 \mathrm{~d} \pm 0.05$ & $3.10 \mathrm{~d} \pm 0.10$ & $2.50 \mathrm{e} \pm 0.05$ & $3.00 \mathrm{e} \pm 0.05$ & $3.03 \mathrm{e} \pm 0.06$ & $3.23 \mathrm{~d} \pm 0.08$ \\
\hline Control & $9.00 \mathrm{a} \pm 0.00$ & $8.87 a \pm 0.08$ & $9.00 \mathrm{a} \pm 0.00$ & $8.97 a \pm 0.03$ & $8.93 a \pm 0.03$ & $9.00 \mathrm{a} \pm 0.00$ & $8.90 \mathrm{a} \pm 0.05$ & $8.93 \mathrm{a} \pm 0.06$ \\
\hline LSD* & 0.65 & 0.77 & 0.54 & 0.35 & 0.51 & 0.41 & 0.39 & 0.65 \\
\hline
\end{tabular}

Means followed by the same letters in each column are not statistically different $(* \mathrm{P}<0.05)$, LSD* $=$ Least significant difference, S.E* $=$ Standard error, $\mu \mathrm{g}$ $\mathrm{ml}^{-1 *}=$ Micro gram per milliliter

Table 6. Effect of different fungicides on mycelial growth of Aspergillus nidulens

\begin{tabular}{|c|c|c|c|c|c|c|c|c|}
\hline \multirow[b]{2}{*}{ Concentration $\left(\mu \mathrm{g} \mathrm{ml}^{-1 *}\right)$} & \multicolumn{7}{|c|}{ Mycelial growth $\left(\mathrm{cm} \pm S . \mathrm{E}^{*}\right)$} & \multirow[b]{2}{*}{ Hexaconazole } \\
\hline & Triton & Alliete & Ridomil Gold & Nativo & Score & Mancozeb & Topsin-M & \\
\hline $01 \mu \mathrm{g} \mathrm{ml}^{-1}$ & $8.83 \mathrm{ab} \pm 0.14$ & $9.00 \mathrm{a} \pm 0.00$ & $8.70 \mathrm{ab} \pm 0.10$ & $8.90 \mathrm{a} \pm 0.05$ & $8.67 \mathrm{a} \pm 0.10$ & $8.83 a \pm 0.06$ & $8.87 \mathrm{a} \pm 0.08$ & $8.70 \mathrm{a} \pm 0.26$ \\
\hline $10 \mu \mathrm{g} \mathrm{ml}^{-1}$ & $8.47 \mathrm{~b} \pm 0.08$ & $8.00 \mathrm{~b} \pm 0.22$ & $8.13 \mathrm{~b} \pm 0.08$ & $8.30 \mathrm{~b} \pm 0.05$ & $7.33 b \pm 0.08$ & $7.60 \mathrm{~b} \pm 0.13$ & $7.33 \mathrm{~b} \pm 0.08$ & $8.50 \mathrm{a} \pm 0.13$ \\
\hline $25 \mu \mathrm{g} \mathrm{ml}^{-1}$ & $7.77 \mathrm{c} \pm 0.08$ & $6.47 c \pm 0.29$ & $7.13 c \pm 0.10$ & $6.93 c \pm 0.19$ & $6.00 \mathrm{c} \pm 0.05$ & $5.93 c \pm 0.23$ & $5.83 c \pm 0.06$ & $7.73 \mathrm{~b} \pm 0.08$ \\
\hline $40 \mu \mathrm{g} \mathrm{ml}^{-1}$ & $6.90 \mathrm{~d} \pm 0.20$ & $5.07 \mathrm{~d} \pm 0.16$ & $5.53 \mathrm{~d} \pm 0.18$ & $5.03 \mathrm{~d} \pm 0.16$ & $4.33 \mathrm{~d} \pm 0.25$ & $4.60 \mathrm{~d} \pm 0.18$ & $4.63 \mathrm{~d} \pm 0.08$ & $6.60 \mathrm{c} \pm 0.15$ \\
\hline $60 \mu \mathrm{g} \mathrm{ml}^{-1}$ & $4.00 \mathrm{e} \pm 0.05$ & $3.93 \mathrm{e} \pm 0.03$ & $3.70 \mathrm{e} \pm 0.35$ & $2.97 \mathrm{e} \pm 0.06$ & $2.83 \mathrm{e} \pm 0.12$ & $3.30 \mathrm{e} \pm 0.18$ & $2.87 \mathrm{e} \pm 0.03$ & $3.13 \mathrm{~d} \pm 0.03$ \\
\hline Control & $8.93 a \pm 0.06$ & $9.00 \mathrm{a} \pm 0.00$ & $8.87 \mathrm{a} \pm 0.08$ & $8.97 a \pm 0.03$ & $9.00 \mathrm{a} \pm 0.00$ & $8.93 a \pm 0.06$ & $9.00 \mathrm{a} \pm 0.00$ & $8.87 \mathrm{a} \pm 0.12$ \\
\hline LSD* & 0.42 & 0.57 & 0.68 & 0.31 & 0.47 & 0.53 & 0.23 & 0.43 \\
\hline
\end{tabular}

Means followed by the same letters in each column are not statistically different $(* \mathrm{P}<0.05)$, LSD ${ }^{*}=$ Least significant difference, S.E* $=$ Standard error, $\mu \mathrm{g}$ $\mathrm{ml}^{-1 *}=$ Micro gram per milliliter 
Table 7. Effect of different fungicides on mycelial growth of Rhizopus stolonifer

\begin{tabular}{|c|c|c|c|c|c|c|c|c|}
\hline \multirow[b]{2}{*}{ Concentration $\left(\mu \mathrm{g} \mathrm{ml}^{-1 *}\right)$} & \multicolumn{7}{|c|}{ Mycelial growth $(\mathrm{cm} \pm$ S.E. $)$} & \multirow[b]{2}{*}{ Hexaconazole } \\
\hline & Triton & Alliete & Ridomil Gold & Nativo & Score & Mancozeb & Topsin-M & \\
\hline $01 \mu \mathrm{g} \mathrm{ml}^{-1}$ & $8.93 a \pm 0.03$ & $8.83 \mathrm{a} \pm 0.14$ & $8.87 a \pm 0.03$ & $8.80 \mathrm{ab} \pm 0.09$ & $8.73 \mathrm{a} \pm 0.06$ & $8.87 \mathrm{a} \pm 0.08$ & $8.83 \mathrm{a} \pm 0.08$ & $8.93 a \pm 0.06$ \\
\hline $10 \mu \mathrm{g} \mathrm{ml}^{-1}$ & $8.57 \mathrm{a} \pm 0.15$ & $8.17 \mathrm{~b} \pm 0.03$ & $8.33 a \pm 0.18$ & $8.50 \mathrm{~b} \pm 0.10$ & $7.37 \mathrm{~b} \pm 0.12$ & $7.57 \mathrm{~b} \pm 0.13$ & $7.53 \mathrm{~b} \pm 0.13$ & $8.47 \mathrm{~b} \pm 0.10$ \\
\hline $25 \mu \mathrm{g} \mathrm{ml}^{-1}$ & $7.50 \mathrm{~b} \pm 0.15$ & $6.43 \mathrm{c} \pm 0.25$ & $7.20 \mathrm{~b} \pm 0.13$ & $7.20 \mathrm{c} \pm 0.26$ & $6.10 \mathrm{c} \pm 0.10$ & $6.37 \mathrm{c} \pm 0.21$ & $6.00 \mathrm{c} \pm 0.13$ & $7.87 \mathrm{c} \pm 0.03$ \\
\hline $40 \mu \mathrm{g} \mathrm{ml}^{-1}$ & $6.73 c \pm 0.03$ & $5.07 \mathrm{~d} \pm 0.08$ & $5.67 \mathrm{c} \pm 0.08$ & $5.33 \mathrm{~d} \pm 0.10$ & $4.40 \mathrm{~d} \pm 0.22$ & $4.73 \mathrm{~d} \pm 0.14$ & $4.93 \mathrm{~d} \pm 0.08$ & $4.50 \mathrm{~d} \pm 0.20$ \\
\hline $60 \mu \mathrm{g} \mathrm{ml}^{-1}$ & $3.53 \mathrm{~d} \pm 0.30$ & $4.00 \mathrm{e} \pm 0.22$ & $3.93 \mathrm{~d} \pm 0.28$ & $3.97 \mathrm{e} \pm 0.06$ & $2.50 \mathrm{e} \pm 0.15$ & $3.70 \mathrm{e} \pm 0.10$ & $3.07 \mathrm{e} \pm 0.28$ & $2.97 \mathrm{e} \pm 0.15$ \\
\hline Control & $9.00 \mathrm{a} \pm 0.00$ & $8.93 \mathrm{a} \pm 0.06$ & $8.83 \mathrm{a} \pm 0.14$ & $9.00 \mathrm{a} \pm 0.00$ & $8.97 \mathrm{a} \pm 0.03$ & $8.93 \mathrm{a} \pm 0.03$ & $8.90 \mathrm{a} \pm 0.09$ & $9.00 \mathrm{a} \pm 0.00$ \\
\hline LSD* & 0.56 & 0.57 & 0.55 & 0.47 & 0.45 & 0.41 & 0.55 & 0.44 \\
\hline
\end{tabular}

Means followed by the same letters in each column are not statistically different $(* \mathrm{P}<0.05)$, LSD* $=$ Least significant difference, S.E* $=$ Standard error, $\mu$ g $\mathrm{ml}^{-1 *}=$ Micro gram per milliliter

Table 8. Effect of different fungicides on mycelial growth of Penicillium

Mycelial growth $\left(\mathrm{cm} \pm \mathrm{S} . \mathrm{E}^{*}\right)$

\begin{tabular}{|c|c|c|c|c|c|c|c|c|}
\hline Concentration $\left(\mu \mathrm{g} \mathrm{ml}^{-1 *}\right)$ & Triton & Alliete & Ridomil Gold & Nativo & Score & Mancozeb & Topsin-M & Hexaconazole \\
\hline $01 \mu \mathrm{g} \mathrm{ml}^{-1}$ & $8.83 a \pm 0.08$ & $8.87 a \pm 0.03$ & $8.90 a \pm 0.09$ & $8.73 a \pm 0.13$ & $8.57 \mathrm{a} \pm 0.06$ & $8.87 a \pm 0.08$ & $8.83 a \pm 0.08$ & $8.83 \mathrm{a} \pm 0.14$ \\
\hline $10 \mu \mathrm{g} \mathrm{ml}^{-1}$ & $8.43 a \pm 0.24$ & $8.30 \mathrm{a} \pm 0.26$ & $8.50 \mathrm{a} \pm 0.26$ & $8.37 \mathrm{a} \pm 0.25$ & $7.33 \mathrm{~b} \pm 0.13$ & $7.63 \mathrm{~b} \pm 0.20$ & $7.77 \mathrm{~b} \pm 0.21$ & $8.30 \mathrm{ab} \pm 0.20$ \\
\hline $25 \mu \mathrm{g} \mathrm{ml}^{-1}$ & $7.13 \mathrm{~b} \pm 0.13$ & $6.63 \mathrm{~b} \pm 0.32$ & $6.97 \mathrm{~b} \pm 0.10$ & $7.03 \mathrm{~b} \pm 0.15$ & $6.03 c \pm 0.12$ & $6.13 \mathrm{c} \pm 0.10$ & $5.73 \mathrm{c} \pm 0.24$ & $7.63 \mathrm{~b} \pm 0.15$ \\
\hline $40 \mu \mathrm{g} \mathrm{ml}^{-1}$ & $5.50 \mathrm{c} \pm 0.26$ & $5.00 \mathrm{c} \pm 0.05$ & $4.10 \mathrm{c} \pm 0.17$ & $5.00 \mathrm{c} \pm 0.09$ & $4.37 \mathrm{~d} \pm 0.25$ & $4.67 \mathrm{~d} \pm 0.26$ & $4.90 \mathrm{~d} \pm 0.30$ & $6.67 \mathrm{c} \pm 0.25$ \\
\hline $60 \mu \mathrm{g} \mathrm{ml}^{-1}$ & $3.57 \mathrm{~d} \pm 0.21$ & $3.93 \mathrm{~d} \pm 0.19$ & $2.37 \mathrm{~d} \pm 0.25$ & $3.60 \mathrm{~d} \pm 0.22$ & $2.63 \mathrm{e} \pm 0.21$ & $3.97 \mathrm{e} \pm 0.18$ & $3.37 \mathrm{e} \pm 0.08$ & $4.00 \mathrm{~d} \pm 0.43$ \\
\hline Control & $8.93 \mathrm{a} \pm 0.06$ & $8.97 \mathrm{a} \pm 0.03$ & $9.00 \mathrm{a} \pm 0.00$ & $8.83 \mathrm{a} \pm 0.08$ & $8.73 \mathrm{a} \pm 0.08$ & $8.93 a \pm 0.06$ & $8.87 \mathrm{a} \pm 0.03$ & $8.86 \mathrm{a} \pm 0.00$ \\
\hline LSD* $^{*}$ & 0.58 & 0.70 & 0.50 & 0.60 & 0.54 & 0.44 & 0.66 & 0.76 \\
\hline
\end{tabular}

Means followed by the same letters in each column are not statistically different $(* \mathrm{P}<0.05)$, LSD* $=$ Least significant difference, S.E* $=$ Standard error, $\mu \mathrm{g}$ $\mathrm{ml}^{-1 *}=$ Micro gram per milliliter

Table 9. Effect of different fungicides on mycelial growth of Drechslera

\begin{tabular}{|c|c|c|c|c|c|c|c|c|}
\hline \multirow[b]{2}{*}{ Concentration $\left(\mu \mathrm{g} \mathrm{ml}^{-1 *}\right)$} & \multicolumn{7}{|c|}{ Mycelial growth $\left(\mathrm{cm} \pm S . E^{*}\right)$} & \multirow[b]{2}{*}{ Hexaconazole } \\
\hline & Triton & Alliete & Ridomil Gold & Nativo & Score & Mancozeb & Topsin-M & \\
\hline $01 \mu \mathrm{g} \mathrm{ml}^{-1}$ & $8.80 \mathrm{a} \pm 0.05$ & $8.77 \mathrm{a} \pm 0.03$ & $8.67 \mathrm{a} \pm 0.14$ & $8.83 a b \pm 0.08$ & $8.53 \mathrm{a} \pm 0.08$ & $8.70 \mathrm{a} \pm 0.05$ & $8.57 \mathrm{a} \pm 0.06$ & $8.93 \mathrm{a} \pm 0.06$ \\
\hline $10 \mu \mathrm{g} \mathrm{ml}^{-1}$ & $8.30 \mathrm{a} \pm 0.20$ & $8.07 \mathrm{~b} \pm 0.08$ & $8.20 \mathrm{a} \pm 0.30$ & $8.37 \mathrm{~b} \pm 0.28$ & $7.43 b \pm 0.29$ & $7.77 \mathrm{~b} \pm 0.19$ & $7.60 \mathrm{~b} \pm 0.23$ & $8.37 \mathrm{ab} \pm 0.23$ \\
\hline $25 \mu \mathrm{g} \mathrm{ml}^{-1}$ & $7.13 \mathrm{~b} \pm 0.10$ & $6.23 \mathrm{c} \pm 0.35$ & $6.80 \mathrm{~b} \pm 0.18$ & $6.83 c \pm 0.15$ & $6.07 \mathrm{c} \pm 0.24$ & $5.97 \mathrm{c} \pm 0.12$ & $5.70 \mathrm{c} \pm 0.22$ & $7.73 b \pm 0.10$ \\
\hline $40 \mu \mathrm{g} \mathrm{ml}^{-1}$ & $6.60 \mathrm{~b} \pm 0.49$ & $5.10 \mathrm{~d} \pm 0.10$ & $5.60 \mathrm{c} \pm 0.28$ & $4.80 \mathrm{~d} \pm 0.18$ & $4.17 \mathrm{~d} \pm 0.15$ & $4.53 \mathrm{~d} \pm 0.33$ & $4.70 \mathrm{~d} \pm 0.26$ & $4.77 \mathrm{c} \pm 0.45$ \\
\hline $60 \mu \mathrm{g} \mathrm{ml}^{-1}$ & $4.47 \mathrm{c} \pm 0.28$ & $4.13 \mathrm{e} \pm 0.23$ & $3.93 \mathrm{~d} \pm 0.14$ & $3.90 \mathrm{e} \pm 0.10$ & $2.50 \mathrm{e} \pm 0.05$ & $3.97 \mathrm{e} \pm 0.08$ & $2.93 \mathrm{e} \pm 0.08$ & $2.87 \mathrm{~d} \pm 0.13$ \\
\hline Control & $8.97 \mathrm{a} \pm 0.03$ & $8.83 \mathrm{a} \pm 0.10$ & $8.70 \mathrm{a} \pm 0.05$ & $8.90 \mathrm{a} \pm 0.05$ & $8.73 \mathrm{a} \pm 0.03$ & $9.00 \mathrm{a} \pm 0.00$ & $8.77 \mathrm{a} \pm 0.08$ & $9.00 \mathrm{a} \pm 0.00$ \\
\hline LSD* & 0.85 & 0.62 & 0.76 & 0.50 & 0.64 & 0.55 & 0.54 & 0.83 \\
\hline
\end{tabular}

Means followed by the same letters in each column are not statistically different $(* \mathrm{P}<0.05)$, LSD* ${ }^{*}$ Least significant difference, S.E* $=$ Standard error, $\mu \mathrm{g}$

$\mathrm{ml}^{-1 *}=$ Micro gram per milliliter 
Table 10. Effect of different fungicides on mycelial growth of Bipolaris

\begin{tabular}{|c|c|c|c|c|c|c|c|c|}
\hline \multirow[b]{2}{*}{ Concentration $\left(\mu \mathrm{g} \mathrm{ml}^{-1 *}\right)$} & \multicolumn{7}{|c|}{ Mycelial growth $\left(\mathrm{cm} \pm \mathrm{S} . \mathrm{E}^{*}\right)$} & \multirow[b]{2}{*}{ Hexaconazole } \\
\hline & Triton & Alliete & Ridomil Gold & Nativo & Score & Mancozeb & Topsin-M & \\
\hline $01 \mu \mathrm{g} \mathrm{ml}^{-1}$ & $8.73 a \pm 0.23$ & $8.90 \mathrm{a} \pm 0.09$ & $8.67 \mathrm{a} \pm 0.08$ & $8.87 \mathrm{a} \pm 0.08$ & $8.63 a \pm 0.16$ & $8.73 a \pm 0.08$ & $8.67 \mathrm{a} \pm 0.16$ & $8.83 \mathrm{a} \pm 0.14$ \\
\hline $10 \mu \mathrm{g} \mathrm{ml}^{-1}$ & $8.03 b \pm 0.20$ & $8.00 \mathrm{~b} \pm 0.09$ & $7.90 \mathrm{~b} \pm 0.30$ & $8.07 b \pm 0.23$ & $7.37 \mathrm{~b} \pm 0.19$ & $7.43 b \pm 0.21$ & $7.30 \mathrm{~b} \pm 0.18$ & $8.23 b \pm 0.25$ \\
\hline $25 \mu \mathrm{g} \mathrm{ml}^{-1}$ & $6.77 \mathrm{c} \pm 0.15$ & $6.07 \mathrm{c} \pm 0.43$ & $6.67 \mathrm{c} \pm 0.25$ & $6.53 c \pm 0.19$ & $5.73 \mathrm{c} \pm 0.25$ & $5.87 \mathrm{c} \pm 0.28$ & $5.20 \mathrm{c} \pm 0.40$ & $7.07 \mathrm{c} \pm 0.08$ \\
\hline $40 \mu \mathrm{g} \mathrm{ml}^{-1}$ & $5.80 \mathrm{~d} \pm 0.18$ & $5.00 \mathrm{~d} \pm 0.18$ & $5.23 \mathrm{~d} \pm 0.21$ & $4.97 \mathrm{~d} \pm 0.06$ & $4.10 \mathrm{~d} \pm 0.10$ & $4.13 d \pm 0.38$ & $4.10 \mathrm{~d} \pm 0.17$ & $6.27 \mathrm{~d} \pm 0.16$ \\
\hline $60 \mu \mathrm{g} \mathrm{ml}^{-1}$ & 4.37 e \pm 0.32 & 3.83 e \pm 0.29 & $2.87 \mathrm{e} \pm 0.03$ & 3.97 e \pm 0.06 & $2.80 \mathrm{e} \pm 0.05$ & $3.50 \mathrm{~d} \pm 0.30$ & $2.67 \mathrm{e} \pm 0.08$ & 4.00 e \pm 0.09 \\
\hline Control & $8.93 \mathrm{a} \pm 0.06$ & $9.00 \mathrm{a} \pm 0.00$ & $8.80 \mathrm{a} \pm 0.10$ & $9.00 \mathrm{a} \pm 0.00$ & $8.83 \mathrm{a} \pm 0.08$ & $9.00 \mathrm{a} \pm 0.00$ & $8.87 \mathrm{a} \pm 0.12$ & $8.97 \mathrm{a} \pm 0.03$ \\
\hline LSD* & 0.62 & 0.74 & 0.65 & 0.45 & 0.61 & 0.90 & 0.80 & 0.48 \\
\hline
\end{tabular}

Means followed by the same letters in each column are not statistically different $(* \mathrm{P}<0.05)$, LSD $^{*}=$ Least significant difference, S.E* $=$ Standard error, $\mu \mathrm{g}$ $\mathrm{ml}^{-1 *}=$ Micro gram per milliliter

\section{Discussion}

Wheat is the major cereal crop of Pakistan and served as staple food for the people. It leads and dominates all crops in production and acreage. Wheat possesses unique characteristics of grains which can be stored for considerable time and changes may occur during transport and storage of these grains. These changes depend on moisture content of wheat grain especially on storage conditions and time duration (Desmarchelier and Ghaly, 1993). Similarly, Chickpea (Cicer arietinum L.) is also an important pulse crop ranked third after dry beans (Phaseolus vulgaris L.) and dry peas (Pisum sativum L.) (Dhar and Gurha, 1998) and Pakistan ranked second in the world in area and third in production (FAO, 2006). This crop is also stored and kept for various seasons in the bags to avoid contamination of several mycoflora which deteriorate the quality of stored gram. Seed borne pathogens are responsible for low germination rate, death of grains, affect seedling vigor and also affect plant morphology (what is the effect of contaminated grains on human health?) (Niaz and Dawar, 2009). The detection of pathogenic seed-borne fungi are an important aspect of disease management. Determining the presence of seed-borne pathogens allow managers to apply the appropriate controls or modify management practices to avoid the problem in the future (Carey et al., 2005). Presence of diseased seeds in seed-lots cannot be reliably detected by visual examination (Kolotelo, 2007). A number of seed borne fungal pathogens were isolated from seed samples of chickpea and wheat. It was observed that Aspergills niger, Aspergillus flavus, Aspergillus sulphureus, Aspergillus nidulense, Rhizopus stolonifer, Penicillium spp. and Drechslera spp. were isolated from wheat seed samples while Aspergillus niger, Aspergillus flavus, Bipolaris spp, Penicillium spp and Rhizopus stolonifer were isolated from seed samples of chickpea. Our results are parallel with Kamal and Mughal (1968) who reported the same mycoflora isolated from wheat samples. Similarly our results regarding the chick pea associated fungi are in accordance with Ali et al., (2010) who observed the similar fungi from the chickpea seeds in artificial media under In-vitro conditions. Seed treatments have the potential to treat seeds therefore, seed treatments should be used only when the gain in germination and seedling survival is greater than the potential loss (Allen et al., 2004). Treating seed as an effort to control seed borne and soilborne diseases has been employed since the middle of the $17^{\text {th }}$ Century. Eight different fungicides namely Triton, Alliete, Ridomil gold, Nativo, Score, Mancozeb, Topsin-M and Hexaconazole were used to determine the mycelial growth of different isolated fungi. Each fungicide were used at the rate of $01 \mu \mathrm{g} \mathrm{ml}^{-1}, 10 \mu \mathrm{g} \mathrm{ml}^{-1}$, $25 \mu \mathrm{g} \mathrm{ml}-1,40 \mu \mathrm{g} \mathrm{ml} \mathrm{gl}^{-1}$ and $60 \mu \mathrm{g}$ 
$\mathrm{ml}^{-1}$ against the poison food technique under in vitro. Score, Topsin-M and Ridomil gold showed significant results at higher doses as compared to the other fungicides treated against the isolated mycoflora. Our results are in line with (Puri, 2013) who showed the same results as found in our study regarding the control of seed borne pathogens isolated from the wheat and chickpea seeds. However, different tested fungicides showed different results against the frequently occurring species of Drechslera and the other rarely occurring fungal species. The out-come of current research also resembles with statements of Bhutta et al., (2001) that score (difenoconazole) gave the best control against fungal pathogens in vitro at 40 to $100 \mu \mathrm{g} \mathrm{ml}^{-1}$. Our results are also parallel with findings of Javaid et al, (2006) that all the fungicides are not significant against seed borne pathogens. Our results are in agreement with results of Walcott et al., (1998) in controlling of seed borne pathogens. Our results are parallel with Pathan et al., (2004) that Topsin-m and Ridomil gold are very effective in controlling of fungal pathogens.

\section{Conclusion}

Stored grains of wheat and chickpea are being polluted under stored conditions through mycoflora. Seed treatment of these grains is necessary to escape the infestation of mycoflora and to obtain a healthy crop stand in the field.

\section{Acknowledgments}

This manuscript is a part of thesis of M. Sc. (Hons.) Plant Pathology of Mr. Majid Karim submitted to the Department of Plant Pathology, Bahauddin Zakariya University, Multan.

\section{References}

Adsule RN, K. S. 1986. Quality of Wheat and Wheat Products. Metropolitan Book Co, New Delhi, India.

Ahmad, I., S. Iftikhar and A. R. Bhutta. 1993. Seed borne microorganism in Pakistan.

Ali, M., M. Khan, A. Rahaman, M. Ahmed and A. Ahsan. 2010. Study on seed quality and performance of some mungbean varieties in Bangladesh. International Journal of Experimental Agriculture, 1: $10-15$.

Allen, T. W., S. A. Enebak and W. A. Carey. 2004. Evaluation of fungicides for control of species of Fusarium on longleaf pine seed. Crop Protection, 23: 979-982.

Belderok, B., J. Mesdag and D. A. Donner. 2000. Manufacturing of other wheat products. Bread- making quality of wheat. Springer Netherlands, pp. 47-54.

Bhutta, A. and S. Hussain. 1999. Seed-borne pathogens of wheat in Pakistan. Barley and Wheat Newsletter.

Bhutta, A. R., M. R. Bhatti, I. Ahmad and I. Sultana. 2001. Chemical control of seed-borne fungal pathogens of sunflower/control químico de patógenos de semillas en girasol/mesures de défense contre les champignons pathogènes du tournesol transmis par la semence. Helia, 24: 67-72.

Bishaw, Z., P. Struik and A. Van Gastel. 2013. Farmer's seed sources and seed quality: 2. Seed health. International Journal of Plant Production, 7: 637657.

Carey, W. A., S. W. Oak and S. A. Enebak. 2005. Pitch canker ratings of longleaf pine clones correlate with Fusarium circinatum infestation of seeds and seedling mortality in containers. Forest Pathology, 35: 205-212.

Desmarchelier, J. M. and T. Ghaly. 1993. Effects of raising the receival moisture content on the storability of Australian wheat. Australian Journal of Experimental Agriculture, 33: 909.

Dhar, V. and S. Gurha. 1998. Integrated Management of chickipea diseases. Integrated pest and disease management. Rajeev, K., Upadhyay, KG, Mukerji, BP, Chamola and Dubey, OP (eds.) APH Pub. Co., New Delhi.(India): 249.

Dharmvir, A., L. Joshi and K. Pathak. 1968. Preliminary note on the occurrence of black point disease of wheat in India. Indian Phytopathology, 21: 234.

Doyer, L. C. 1938. Manual for the determination of seedborne diseases. Manual for the determination of seed-borne diseases.

FAO 2007. GIEWS workstation. Available at: http://lprapp08. fao.org/fenix-portal (Accessed 5 November 2007

Fakir, G. 1999. Seed Health-an Indispensable Agrotechnology for crop production. Lecture note for course on Agro-technology and Environment Management for the CARITAS officers at GTI, BAU, Mymensingh from: 1-4.

Hulse, J. H. 1994. Nature, composition, and utilization of food legumes. Expanding the Production and Use of Cool Season Food Legumes. Springer Netherlands, pp. 77-97.

Javaid, A., A. Ashraf, N. Akhtar, M. Hanif and M. A. Farooq. 2006. Efficacy of some fungicides against seed- 
borne mycoflora of wheat. Journal of Mycophytopathology, 4: 45-49.

Joshi, M. M. a. R. S. S. A. 1969. Botrytis grey mould of gram. Indian Phytopathology, 22: 125-128.

Kamal, M. and S. M. Moghal. 1968. Studies on plant diseases of South West Pakistan. Studies on plant diseases of South West Pakistan.

Khan, H., S. Iqbal, A. Haqqani, S. Khan and B. Malik. 1991. Thal-the home of chickpea in Pakistan. International Chickpea Newsletter, 24: 7-10.

Kolotelo, D., Steenis, E. V., Peterson, M., Bennet, R., Trotter, D. and Dennis, J. 2007. Seed Handling Guidebook, Canada.

Kumar, A., U. S. Singh, J. Kumar and G. K. Garg. 2008. Application of molecular and immuno-diagnostic tools for detection, surveillance and quarantine regulation of Karnal bunt (Tilletia indica) of wheat. Food and Agricultural Immunology, 19: 293-311.

Nene, Y. L. a. P. N. T. 1980. Fungicides in Plant Disease Control. Oxford and IBH Publishing House, New Delhi.

Niaz, I. and S. Dawar. 2009. Detection of seed borne mycoflora in maize (Zea mays L.). Pakistan Journal of Botany, 41: 443-451.

Pathan, M. A., M.M. Jiskani, K.H. Wagan and M. Imran. 2004. Efficacy of different seed dressing fungicides on seed rotting fungi and growth of tomato plants. Pakistan Journal of Seed Technology, 1: 16-31.

Puri, K. D. 2013. Comparative Population Genetics of Fusarium Graminearum and Novel Sources of Resistance to Fusarium Head Blight in Spring Wheat. North Dakota State University.

Ryley, M. J., H. F. Mosetter and J. L. Rose. 1989. Yield losses of soybeans due to Phytophthora megasperma f.sp. Glycinea. Australian Journal of Agricultural Research, 40: 1161.

Simmonds, N. W. 1986. Grain Legume Crops. Edited by R. J. Summerfield and E. H. Roberts. London: Collins 1985, pp. 859, £30.00. Experimental Agriculture, 22: 314 .

Sinclair, J. B. and V. K. Agarwal. 1997. Principles of Seed Pathology. CRC Press.

Vishwakarma, S. N. and K. C. B. Chaudhary. 1974. Floral blight of gram incited by Alternaria alternata. Netherlands Journal of Plant Pathology, 80: 110112.

Walcott, R. R., D. C. McGee and M. K. Misra. 1998. Detection of Asymptomatic Fungal Infections of Soybean Seeds by Ultrasound Analysis. Plant Disease, 82: 584-589.

Wiese, M. V. 1984. Compendium of wheat diseases. 3 ed. The American Phytopathological Society. 